Supporting Information

\title{
Effects of AuCuB Catalysts with Porous Nanostructures on Electrosynthesis of
}

\section{Ammonia}

Ziqiang Wang, Jinhua Niu, You Xu, Liang Wang, Hongjing Wang and Huazhang Liu*

State Key Laboratory Breeding Base of Green-Chemical Synthesis Technology, College of Chemical Engineering, Zhejiang University of Technology, Hangzhou 310014, P.R. China.

*Corresponding author's E-mail: cuihua@zjut.edu.cn

Number of pages: 10

Number of figures: 11

Number of tables: 1 

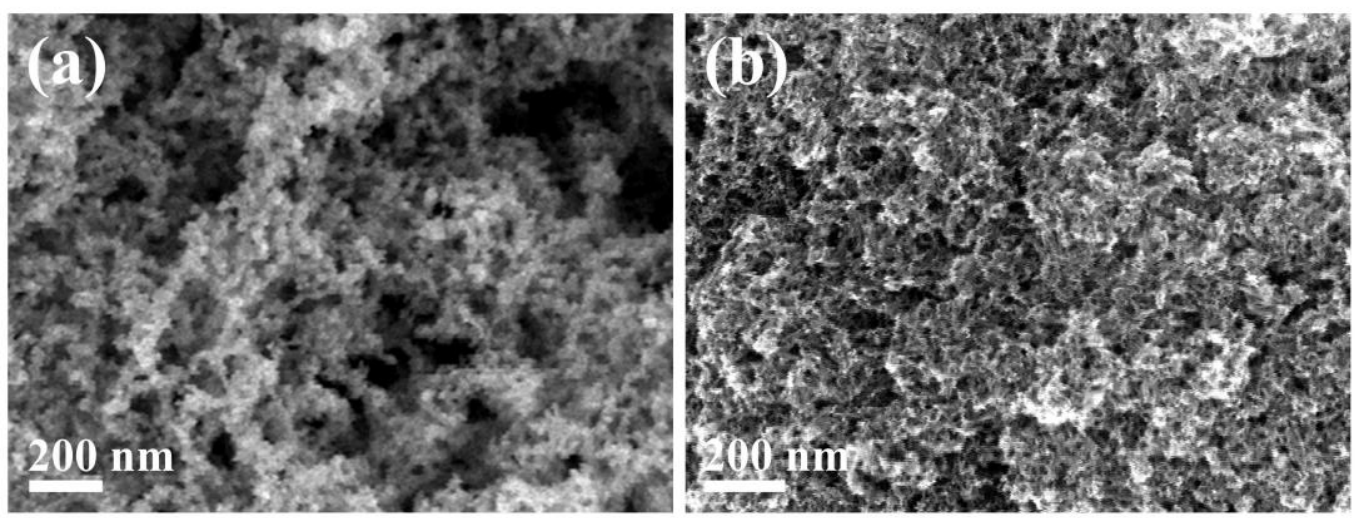

Figure S1. SEM images of the (a) AuCuB PNSs and (b) AuCu PNSs.
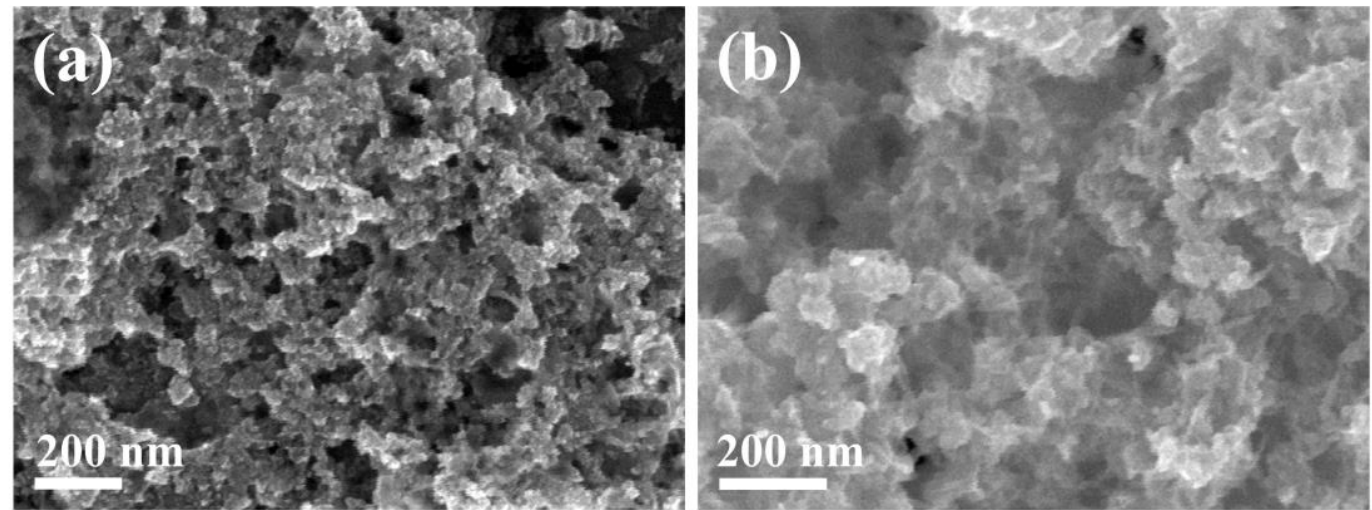

Figure S2. SEM images of the samples prepared with different concentrations of $\mathrm{NaBH}_{4}$ : (a) 10 $\mathrm{mM}$ and (b) $50 \mathrm{mM}$. 

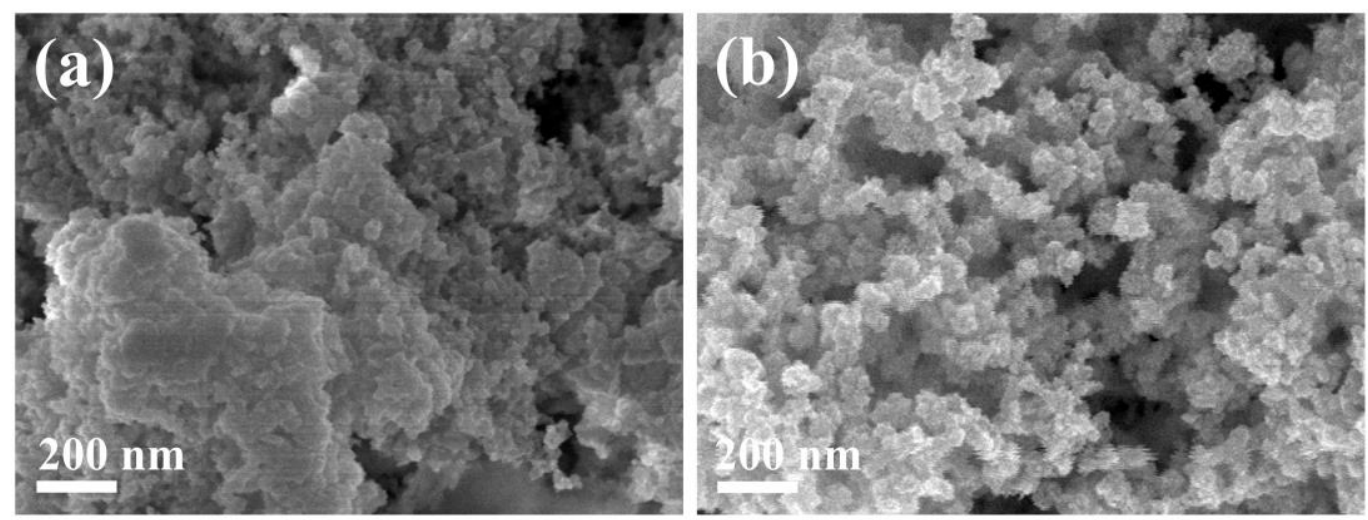

Figure S3. SEM images of the samples prepared with different amounts of reducing agent and metallic precursors: (a) $\mathrm{NaBH}_{4}(4 \mathrm{~mL}, 20 \mathrm{mM}), \mathrm{CuCl}_{2}(2 \mathrm{~mL}, 20 \mathrm{mM}), \mathrm{HAuCl}_{4}(2 \mathrm{~mL}, 20 \mathrm{mM})$; (b) $\mathrm{NaBH}_{4}(40 \mathrm{~mL}, 20 \mathrm{mM}), \mathrm{CuCl}_{2}(2 \mathrm{~mL}, 20 \mathrm{mM}), \mathrm{HAuCl}_{4}(2 \mathrm{~mL}, 20 \mathrm{mM})$.
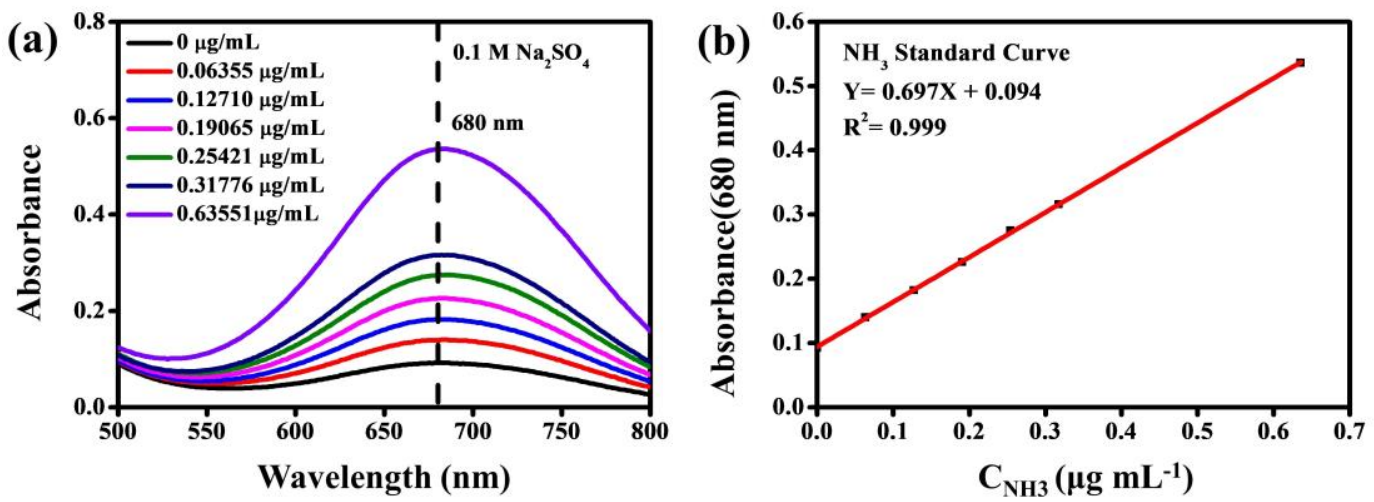

Figure S4. (a) UV-vis absorption spectra of indophenol assays with $\mathrm{NH}_{3}$ in $0.1 \mathrm{M} \mathrm{Na}_{2} \mathrm{SO}_{4}$ after incubated for $1 \mathrm{~h}$ at room temperature. (b) Calibration curve used for calculation of $\mathrm{NH}_{3}$ concentration. The fitting curve shows good linear relation of absorbance with $\mathrm{NH}_{3}$ concentrations $\left(\mathrm{Y}=0.697 \mathrm{X}+0.094, \mathrm{R}^{2}=0.999\right)$ 

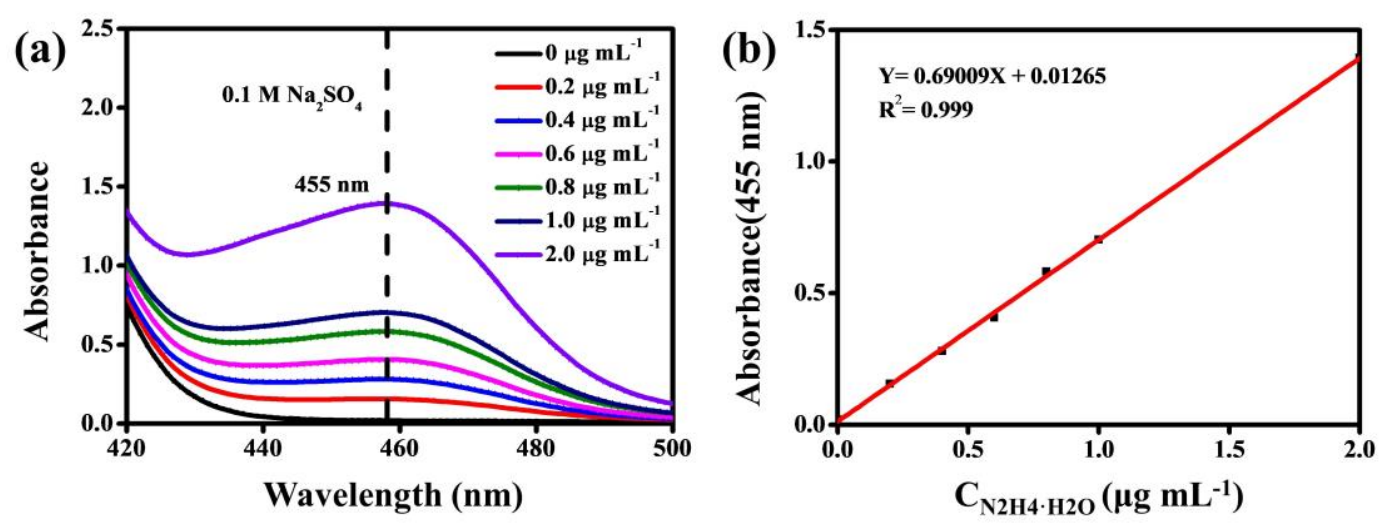

Figure S5. (a) UV-vis absorption spectra of various $\mathrm{N}_{2} \mathrm{H}_{4} \cdot \mathrm{H}_{2} \mathrm{O}$ concentrations after incubated for 15 min at room temperature. (b) Calibration curve used for calculation of $\mathrm{N}_{2} \mathrm{H}_{4} \cdot \mathrm{H}_{2} \mathrm{O}$ concentrations. The fitting curve shows good linear relation of absorbance with $\mathrm{N}_{2} \mathrm{H}_{4} \cdot \mathrm{H}_{2} \mathrm{O}$ concentrations $(\mathrm{Y}=$ $\left.0.69009 X+0.01265, R^{2}=0.999\right)$

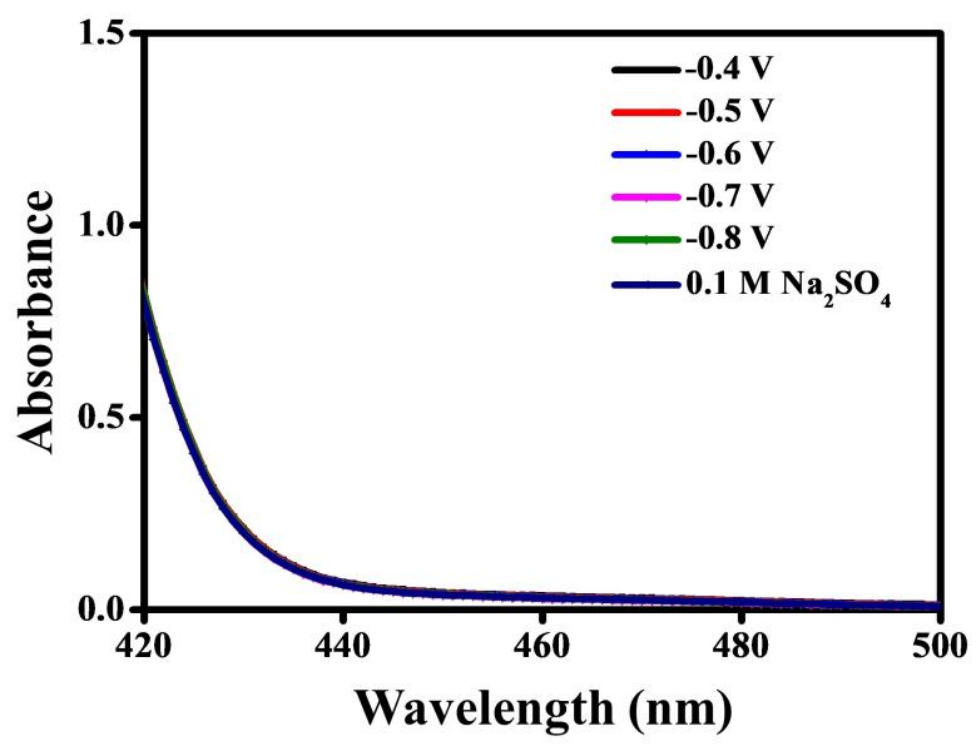

Figure S6. UV-vis absorption spectra of different electrolysis solutions with color development. 


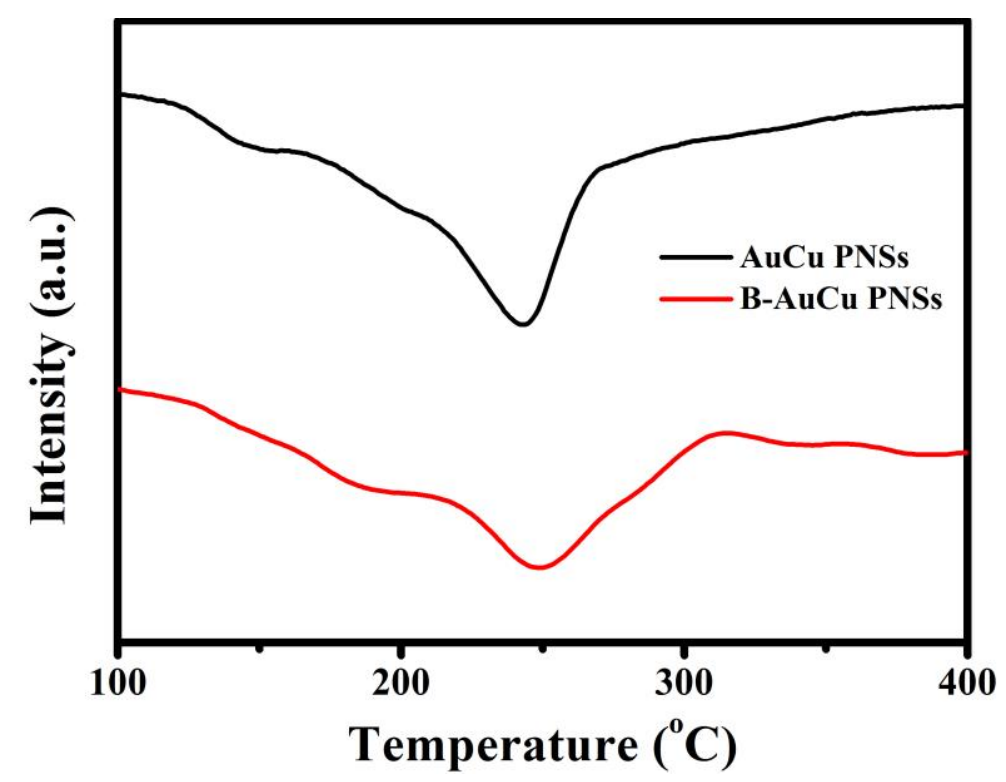

Figure S7. $\mathrm{N}_{2}$-TPD profiles of AuCu PNSs and AuCuB PNSs.
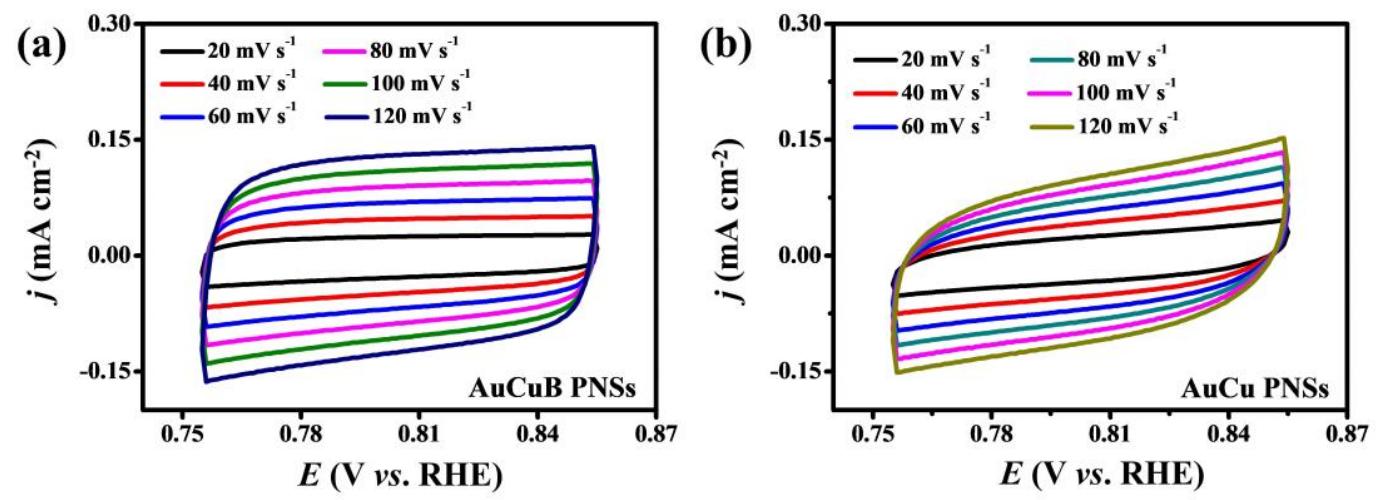

Figure S8. CVs of (a) AuCuB PNSs and (b) AuCu PNSs with various scan rates between 20 and $120 \mathrm{mV} \mathrm{s}^{-1}$ in the 0.756 to $0.856 \mathrm{~V}$. 


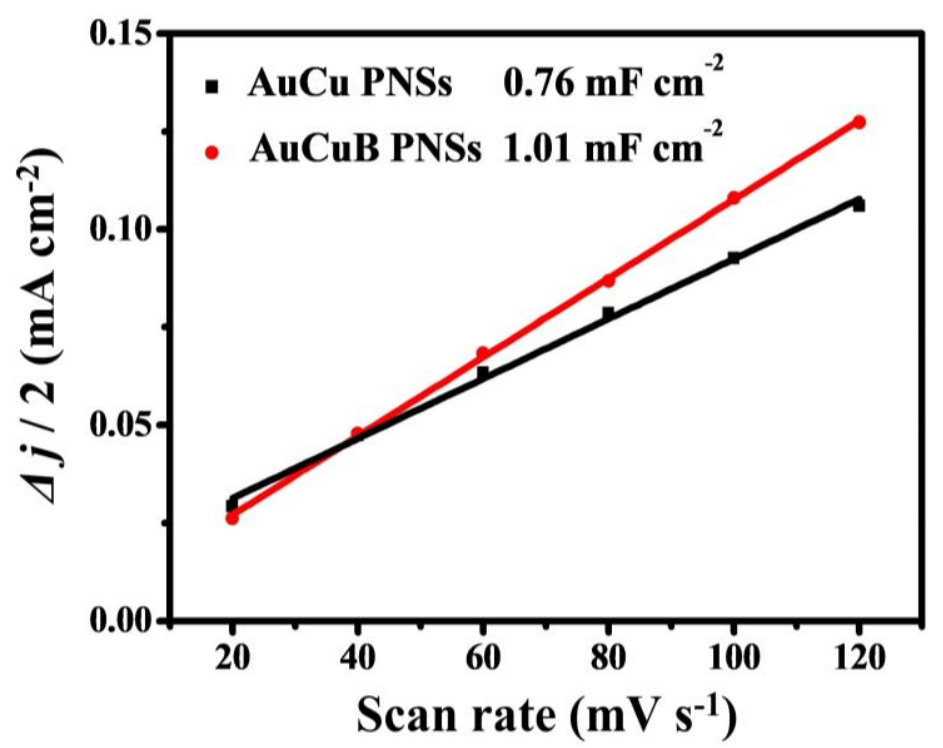

Figure S9. The capacitive currents at $0.806 \mathrm{~V}$ as a function of scan rate for $\mathrm{AuCuB}$ PNSs and $\mathrm{AuCu}$ PNSs.

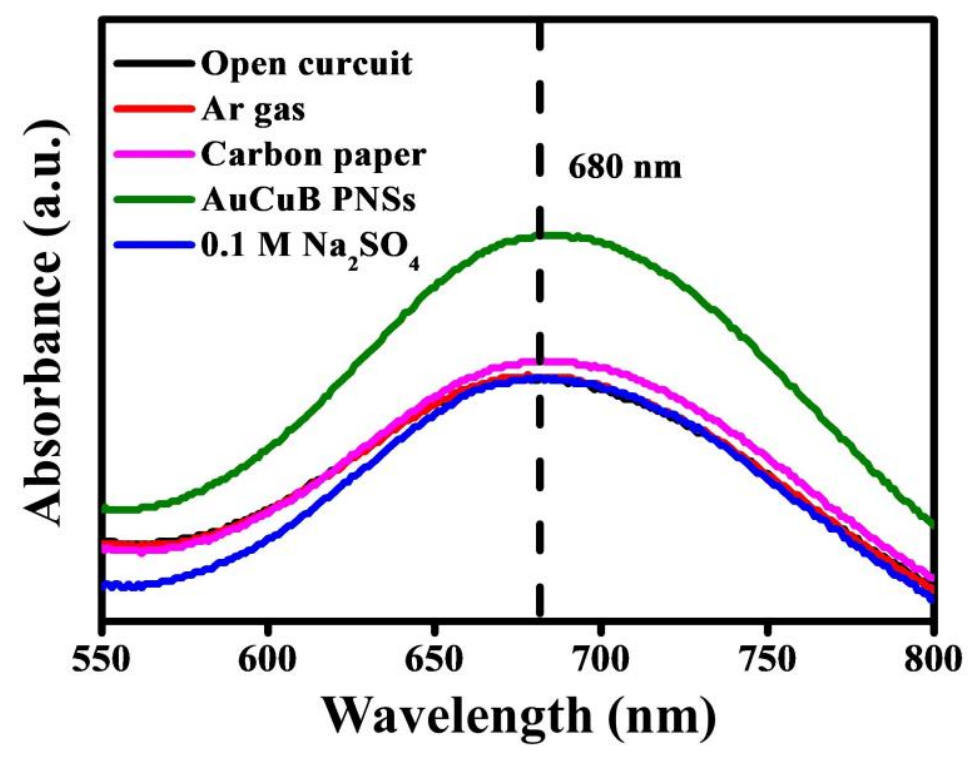

Figure S10. UV-vis absorption spectra of electrolysis solutions stained with indophenol indicator under different electrochemical conditions. 

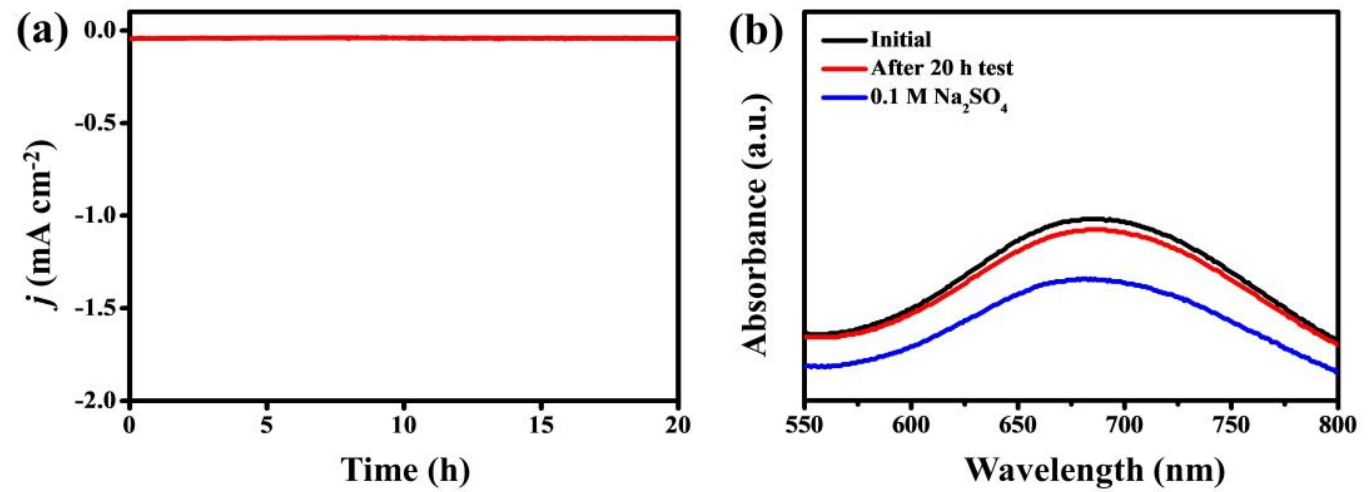

Figure S11. (a) Chronoamperometric curve of AuCuB PNSs after long-term durability measurement at $-0.5 \mathrm{~V}$ for $20 \mathrm{~h}$ and (b) corresponding $\mathrm{UV}$-vis absorption spectra of the electrolytes before and after electrolysis. 
Table S1. Comparison of the electrocatalytic $\mathrm{N}_{2}$ reduction performance for AuCuB PNSs with some previously reported NRR electrocatalysts at ambient conditions.

\begin{tabular}{|c|c|c|c|c|}
\hline Catalysts & Electrolyte & $\begin{array}{c}\mathrm{NH}_{3} \text { yield } \\
\text { ( } \mu \mathrm{g} \mathrm{h}^{-1} \mathrm{mg}^{-1} \text { cat.) }\end{array}$ & $\begin{array}{l}\mathrm{FE} \\
(\%)\end{array}$ & Ref. \\
\hline AuCuB PNSs & $0.1 \mathrm{M} \mathrm{Na}_{2} \mathrm{SO}_{4}$ & 13.20 & 12.78 & This work \\
\hline$\gamma-\mathrm{Fe}_{2} \mathrm{O}_{3}$ & $0.1 \mathrm{M} \mathrm{KOH}$ & 0.21 & 1.9 & {$[1]$} \\
\hline Nanoporous $\mathrm{Pd}_{1} \mathrm{Ag}_{1}$ & $1 \mathrm{M} \mathrm{KOH}$ & 24.1 & $\sim 1.7$ & {$[2]$} \\
\hline $\mathrm{Mn}_{3} \mathrm{O}_{4}$ nanocube & $0.1 \mathrm{M} \mathrm{Na}_{2} \mathrm{SO}_{4}$ & 11.6 & 3.00 & {$[3]$} \\
\hline Au nanorods & $0.1 \mathrm{M} \mathrm{KOH}$ & 6.04 & 4 & {$[4]$} \\
\hline PdRu tripods & $0.1 \mathrm{M} \mathrm{KOH}$ & 37.23 & 1.85 & {$[5]$} \\
\hline$\alpha-\mathrm{Au} / \mathrm{CeO} \mathrm{x}-\mathrm{RGO}$ & $0.1 \mathrm{M} \mathrm{HCl}$ & 8.31 & 10.10 & {$[6]$} \\
\hline PCN-NV4 & $0.1 \mathrm{M} \mathrm{HCl}$ & 8.09 & 11.59 & [7] \\
\hline $\mathrm{Pd} / \mathrm{C}$ & $0.1 \mathrm{M}$ PBS & 4.5 & 8.2 & [8] \\
\hline $\mathrm{Fe}_{2} \mathrm{O}_{3}$ nanorods & $0.1 \mathrm{M} \mathrm{Na}_{2} \mathrm{SO}_{4}$ & 15.9 & 0.94 & [9] \\
\hline $\mathrm{MoO}_{3}$ & $0.1 \mathrm{M} \mathrm{HCl}$ & 29.43 & 1.9 & {$[10]$} \\
\hline $\mathrm{Mo}_{2} \mathrm{~N}$ & $0.1 \mathrm{M} \mathrm{HCl}$ & 78.4 & 4.5 & {$[11]$} \\
\hline Au flowers & $0.1 \mathrm{M} \mathrm{HCl}$ & 25.57 & 6.05 & {$[12]$} \\
\hline Boron-doped $\mathrm{TiO}_{2}$ & $0.1 \mathrm{M} \mathrm{Na}_{2} \mathrm{SO}_{4}$ & 14.4 & 3.4 & {$[13]$} \\
\hline
\end{tabular}




\section{References}

(1) Kong, J.; Lim, A.; Yoon, C.; Jang, J. H.; Ham, H. C.; Han, J.; Nam, S.; Kim D.; Sung, Y. E., Choi, J.; Park, H. S. Electrochemical Synthesis of $\mathrm{NH}_{3}$ at Low Temperature and Atmospheric Pressure Using a $\gamma-\mathrm{Fe}_{2} \mathrm{O}_{3}$ Catalyst. ACS Sustainable Chem. Eng. 2017, 5 (11), 10986-10995, DOI: $10.1021 /$ acssuschemeng.7b02890.

(2) Pang, F. J.; Wang, F.; Yang, L. T.; Wang, Z. F.; Zhang, W. Q. Hierarchical Nanoporous Pd $_{1} \operatorname{Ag}_{1}$ Alloy Enables Efficient Electrocatalytic Nitrogen Reduction under Ambient Conditions. Chem. Commun. 2019, 55 (68), 10108-10111, DOI: 10.1039/C9CC04460D.

(3) Wu, X. F.; Xia, L.; Wang, Y.; Lu W. B.; Liu, Q.; Shi, X. F.; Sun, X. P. $\mathrm{Mn}_{3} \mathrm{O}_{4}$ Nanocube: An Efficient Electrocatalyst toward Artificial $\mathrm{N}_{2}$ Fixation to $\mathrm{NH}_{3}$. Small 2018, 14 (48), 1803111-1803116, DOI: 10.1002/smll.201803111.

(4) Bao, D.; Zhang, Q.; Meng, F.-L.; Zhong, H.-X.; Shi, M.-M.; Zhang, Y.; Yan, J.-M.; Jiang, Q.; Zhang, X.-B. Electrochemical Reduction of $\mathrm{N}_{2}$ under Ambient Conditions for Artificial $\mathrm{N}_{2}$ Fixation and Renewable Energy Storage Using $\mathrm{N}_{2} / \mathrm{NH}_{3}$ Cycle. Adv. Mater. 2017, 29 (3), 1604799, DOI: 10.1002/adma.201604799.

(5) Wang, H.; Li, Y.; Li, C.; Deng, K.; Wang, Z.; Xu, Y.; Li, X.; Xue, H.; Wang, L. One-Pot Synthesis of Bi-Metallic PdRu Tripods as an Efficient Catalyst for Electrocatalytic Nitrogen Reduction to Ammonia. J. Mater. Chem. A 2019, 7 (2), 801-805, DOI: 10.1039/C8TA09482A.

(6) Li, S. J.; Bao, D.; Shi, M. M.; Wulan, B. R.; Yan, J. M.; Jiang, Q. Amorphizing of Au Nanoparticles by $\mathrm{CeO}_{x}-\mathrm{RGO}$ Hybrid Support towards Highly Efficient Electrocatalyst for $\mathrm{N}_{2}$ Reduction under Ambient Conditions. Adv. Mater. 2017, 29 (33), 1700001-1700006, DOI: 10.1002/adma.201700001.

(7) Lv, C.; Qian, Y. M.; Yan, C. S.; Ding, Y.; Liu, Y. Y.; Chen, G.; Yu, G. H. Defect Engineering Metal-Free Polymeric Carbon Nitride Electrocatalyst for Effective Nitrogen Fixation under Ambient Conditions. Angew. Chem., Int. Ed. 2018, 57 (32), 10246-10250, DOI: 10.1002/anie.201806386.

(8) Wang, J.; Yu, L.; Hu, L.; Chen, G.; Xin, H. L.; Feng, X. F. Ambient Ammonia Synthesis via Palladium-Catalyzed Electrohydrogenation of Dinitrogen at Low Overpotential. Nat. Commun. 2018, 9 (1), 1795-1801, DOI: 10.1038/s41467-018-04213-9. 
(9) Xiang, X.; Wang, Z.; Shi, X.; Fan, M.; Sun, X. Ammonia Synthesis from Electrocatalytic $\mathrm{N}_{2}$ Reduction under Ambient Conditions by $\mathrm{Fe}_{2} \mathrm{O}_{3}$ Nanorods. ChemCatChem 2018, 10 (20), 4530-4535, DOI: $10.1002 /$ cctc.201801208.

(10) Han, J.; Ji, X.; Ren, X.; Cui, G.; Li, Lei.; Xie, F.; Wang, H.; Li, B.; Sun, X. MoO $\mathrm{M}_{3}$ Nanosheets for Efficient Electrocatalytic $\mathrm{N}_{2}$ Fixation to $\mathrm{NH}_{3}$. J. Mater. Chem. A 2018, 6 (27), 12974-12977, DOI: $10.1039 / \mathrm{C} 8 \mathrm{TA} 03974 \mathrm{G}$.

(11) Ren, X.; Cui, G.; Chen, L.; Xie, F.; Wei, Q.; Tian, Z.; Sun, X. Electrochemical N2 Fixation to $\mathrm{NH}_{3}$ under Ambient Conditions: $\mathrm{Mo}_{2} \mathrm{~N}$ Nanorod as a Highly Efficient and Selective Catalyst. Chem. Commun. 2018, 54 (61), 8474-8477, DOI: 10.1039/C8CC03627F.

(12) Wang, Z.; Li, Y.; Yu, H.; Xu, Y.; Xue, H.; Li, X.; Wang, H.; Wang, L. Ambient Electrochemical Synthesis of Ammonia from Nitrogen and Water Catalyzed by Flower-Like Gold Microstructures. ChemSusChem 2018, 11 (19), 3480-3485, DOI: 10.1002/cssc.201801444.

(13) Wang, Y.; Jia, K.; Pan, Q.; Xu, Y.; Liu, Q.; Cui, G.; Guo, X.; Sun, X. Boron-Doped $\mathrm{TiO}_{2}$ for Efficient Electrocatalytic $\mathrm{N}_{2}$ Fixation to $\mathrm{NH}_{3}$ at Ambient Conditions. ACS Sustainable Chem. Eng. 2018, 7 (1), 117-122, DOI: 10.1021/acssuschemeng.8b05332. 